\title{
Synthesis, Properties, and Applications of Polymeric Nanocomposites
}

\author{
Sevan P. Davtyan, ${ }^{1}$ Aleksandr Berlin, ${ }^{2}$ Vladimir Agabekov, ${ }^{3}$ and Nodari Lekishvilii ${ }^{4}$ \\ ${ }^{1}$ State Engineering University of Armenia, 105 Teryan Street, 375009 Yerevan, Armenia \\ ${ }^{2}$ Semenov Institute of Chemical Physics, Russian Academy of Sciences, 4 Kosygin Street, Moscow 119991, Russia \\ ${ }^{3}$ Institute of New Materials Chemistry, National Academy of Sciences of Belarus, 36 Fr. Skorini, 220141 Minsk, Belarus \\ ${ }^{4}$ Ivane Javakhishvili Tbilisi State University, Ilia Chavchavadze Avenue, 0179 Tbilisi, Georgia \\ Correspondence should be addressed to Sevan P. Davtyan, davtyans@seua.am
}

Received 19 June 2012; Accepted 19 June 2012

Copyright (C) 2012 Sevan P. Davtyan et al. This is an open access article distributed under the Creative Commons Attribution License, which permits unrestricted use, distribution, and reproduction in any medium, provided the original work is properly cited.

For the current special issue of this journal were received 49 manuscripts from them 6 have been withdrawn, 7-rejected, and 35-accepted. The geometry of papers rather wide: USA, México, Chile, France, Germany, UK, Italy, Spain, Czech Republic, Poland, Romania, Slovenia, Republic of Belarus, Turkey, Saudi Arabia, China, Korea, Taiwan, Malaysia, Viet Nam.

Two reviews "Synthesis of silica nanoparticles by sol-gel: size-dependent properties, surface modification, and applications in silica-polymer nanocomposites - a review," and "Polymer/QDs nanocomposites for waveguiding applications."

In the article "Synthesis of silica nanoparticles by sol-gel: size-dependent properties, surface modification, and applications in silica-polymer nanocomposites - a review," the literature data on $\mathrm{SiO}_{2}$ nanoparticles obtainment and polymer nanocomposites synthesis by sol-gel method was considered, the possibilities of obtained product's properties regulation and their application are analyzed.

At the review "Polymer/QDs nanocomposites for waveguiding applications," were considered the possibilities of Active wave guides with use of Quantum Points CdTe and PMMA binding_polymer/QDs nanocomposites for waveguiding on hybrid organic (polymer) -inorganic (quantum dots) materials applications.

The "Bragg grating optical filters by UV nanoimprinting," "Polymer/QDs nanocomposites for waveguiding applications," "Polyol-mediated synthesis of zinc oxide nanorods and nanocomposites with poly(methyl methacrylate)," "Matrix-assisted pulsed laser thin film deposition by using Nd:YAG laser," "Introduction of bifunctional group onto MWNT by radiationinduced graft polymerization and its use as biosensorsupporting materials," "Evaluation of CdS interfacial layers in $\mathrm{ZnO}$ nanowire/poly(3-hexylthiophene) solar cells," and "Materials and devices research of PPV-ZnO nanowires for heterojunction solar cells," completely correspond to the title of current special issue. The articles are devoted to the various applications. For example were obtained the following: in "Bragg grating optical filters by UV nanoimprinting," the stable micro- and nanostructured optical devices, in "Polymer/QDs nanocomposites for waveguiding applications," nanocomposites for wave-guiding, in "Introduction of bifunctional group onto MWNT by radiation-induced graft polymerization and its use as biosensor-supporting materials," biosensor for the detection of phenolic compounds in drinks such as red wine and juices and the zinc oxide nanowires, used directly as negative electrode materials for photovoltaic devices as well, and so on.

The following 26 papers can be conventionally divided to the four groups.

To the first group can be attributed the articles related to carbon-contained (nanotubes: "An electrically conductive and organic solvent vapors detecting composite composed of an entangled network of carbon nanotubes embedded in polystyrene," "Synthesis and characterizations of poly(3hexylthiophene) and modified carbon nanotube composites, "Temperature dependence of mechanical and tribological 
performance of MWNTfilled short fibre reinforced multiscale phenolic friction composites") morphology, microstructure and physical properties, "Cast nanostructured films of poly(methyl methacrylate-b-butyl acrylate)/carbon nanotubes: influence of poly(butyl acrylate) content on film evaporation rate, morphology, and electrical resistance," fullerenes $\mathrm{C}_{60}-$ "Effect of fullerene derivates on thermal and crystallization behavior of PBT/decylamine-C 60 and PBT/TCNEO$C{ }_{60}$ nanocomposites,"-influence of various concentration fullerene on thermal properties nanocomposites, carbon additives (carbon fillers) - "Effect of nanodisperse carbon fillers and isocyanate chain extender on structure and properties of poly(ethylene terephthalate),"-mechanical, rheological, relaxation properties and on structure are investigated, graphite- "Simple method for the preparation of Composites Based on PA6 and partially exfoliated graphite,"-polymer nanocomposites with various binding and carbon/carbon composites. In the specified works are investigated physicmechanical "An electrically conductive and organic solvent vapors detecting composite composed of an entangled network of carbon nanotubes embedded in polystyrene," "Synthesis and characterizations of poly(3-hexylthiophene) and modified carbon nanotube composites," "Effect of nanodisperse carbon fillers and isocyanate chain extender on structure and properties of poly (ethylene terephthalate), "relaxation "Effect of nanodisperse carbon fillers and isocyanate chain extender on structure and properties of poly(ethylene terephthalate), " rheological "Effect of nanodisperse carbon fillers and isocyanate chain extender on structure and properties of poly(ethylene terephthalate)," thermal "Effect of fullerene derivates on thermal and crystallization behavior of PBT/decylamine-C 60 and PBT/TCNEO-C 60 nanocomposites," and electric properties.

To the second group it is possible to attribute the 9 articles were investigated nanoadditives: metallic (silver: "Photogenerating silver nanoparticles and polymer nanocomposites by direct activation in the near infrared," "Optical properties of polyvinyl alcohol films modified with silver nanoparticles," Copper: "Electrical and thermal behavior of copper-epoxy nanocomposites prepared via aqueous to organic phase transfer technique") and metallic oxides $\left(\mathrm{Fe}_{3} \mathrm{O}_{4}\right.$ : "Nanocomposites based on technical polymers and sterically functionalized soft magnetic magnetite nanoparticles: synthesis, processing, and characterization," "Fabrication and characterization of magnetoresponsive electrospun nanocomposite membranes based on methacrylic random copolymers and magnetite nanoparticles," $\mathrm{SiO}_{2}$ : "Study on the compressive modulus of nylon-11/silica nanocomposites," "Polypropylene nanocomposites obtained by in situ polymerization using metallocene catalyst: influence of the nanoparticles on the final polymer morphology," $\mathrm{TiO}_{2}$ : "The effect of film thickness and $\mathrm{TiO}_{2}$ content on film formation from $\mathrm{PS} / \mathrm{TiO}_{2}$ nanocomposites prepared by dip-coating method," $\mathrm{Al}_{2} \mathrm{O}_{3}$ : "Microstructure and mechanical properties of heterogeneous ceramic-polymer composite using interpenetrating network"). In the works were used nontraditional methods of polymer composites obtainment-"Photogenerating silver nanoparticles and polymer nanocomposites by direct activation in the near infrared," polymethine dyes were used as the photoactive agents the present paper with the photochemically assisted fabrication of silver nanoparticles in a polymerizable medium using a near infrared (NIR) source. "Optical properties of polyvinyl alcohol films modified with silver nanoparticles," is examine the ability of PVA films modified with silver nanoparticles, to scatter incident linear polarized He-Ne laser light, as well as were determined the effect of processing conditions (uniaxial tension, crosslinking of the polymer matrix during irradiation by UV radiation in presence of bichromates) on scattering and polarizing properties of modified PVA films.

In "Polypropylene nanocomposites obtained by in situ polymerization using metallocene catalyst: influence of the nanoparticles on the final polymer morphology" were used in situ polymerizations of polypropylene obtainment by two methods: (1) the nanoparticles were added together with the catalytic system (rac-5 $\mathrm{Et}(\mathrm{Ind}){ }_{2} \mathrm{ZrCl}_{2} /(\mathrm{MAO})$ directly into the reactor, (2) the catalytic system puts on $\mathrm{SiO}_{2}$ surface and entered into the reactor the metallocene rac-Et(Ind) ${ }_{2} \mathrm{ZrCl}_{2}$ was supported on silica nanospheres pretreated with.

In "Electrical and thermal behavior of copper-epoxy nanocomposites prepared via aqueous to organic phase transfer technique," using transfer technique were received the Epoxy resin/Copper nanocomposites.

Thus, in works "Photogenerating silver nanoparticles and polymer nanocomposites by direct activation in the near infrared," "Optical properties of polyvinyl alcohol films modified with silver nanoparticles," "Electrical and thermal behavior of copper-epoxy nanocomposites prepared via aqueous to organic phase transfer technique," as well as in "Nanocomposites based on technical polymers and sterically functionalized soft magnetic magnetite nanoparticles: synthesis, processing, and characterization," "Fabrication and characterization of magnetoresponsive electrospun nanocomposite membranes based on methacrylic random copolymers and magnetite nanoparticles," "Study on the compressive modulus of nylon-11/silica nanocomposites," "Polypropylene nanocomposites obtained by in situ polymerization using metallocene catalyst: influence of the nanoparticles on the final polymer morphology," "The effect of film thickness and $\mathrm{TiO}_{2}$ content on film formation from PS/ $\mathrm{TiO}_{2}$ nanocomposites prepared by dip-coating method," and "Microstructure and mechanical properties of heterogeneous ceramic-polymer composite using interpenetrating network," using nontraditional methods of nanocomposites synthesis it was shown the improvement dynamical-mechanical "Nanocomposites based on technical polymers and sterically functionalized soft magnetic magnetite nanoparticles: synthesis, processing, and characterization," magnetic passableness supermagnetic behaviour "Fabrication and characterization of magnetoresponsive electrospun nanocomposite membranes based on methacrylic random copolymers and magnetite nanoparticles," of polymer nanocomposites with the various binders.

In the current special issue were accepted the papers "Study structure and properties of nanocomposite material based on unsaturated polyester with clay modified by poly(ethylene oxide)," "PP/MMT nanocomposite: mathematic modelling of layered nanofiller," "Role of polymer interactions with clays and modifiers on mechanical properties and crystallinity in polymer clay nanocomposites," and "The effect of nanoclay on dust generation during drilling of pa6 
nanocomposites," on polymer/clay nanocomposites. In "Study structure and properties of nanocomposite material based on unsaturated polyester with clay modified by poly(ethylene oxide)," the authors have tried using Poly(ethylene oxide) as a new modifier to replace some traditional ionic surfactants, а в "Role of polymer interactions with clays and modifiers on nanomechanical properties and crystallinity in polymer clay nanocomposites," describes a quantitative study of interaction energies on properties of nanocomposites. In the article "The effect of nanoclay on dust generation during drilling of pa6 nanocomposites," by nanoclay particles filled polyamide 6 nanocomposites during mechanical drilling were obtained. Mathematic modeling "PP/MMT nanocomposite: mathematic modelling of layered nanofiller," of layered nanofiller $\mathrm{PP} / \mathrm{MMT}$ nanocomposite was carried out.

In "The low-temperature crystallization and interface characteristics of $\mathrm{ZnInSnO} / \mathrm{in}$ films using a biascrystallization mechanism," "Preparation and characterization of folate targeting magnetic nanomedicine loaded with cisplatin," and "Biodegradable polymer-coated, gelatin hydrogel/bioceramics ternary composites for antitubercular drug delivery and tissue regeneration" were investigated: influence of heat on the crystallization process of $\mathrm{ZnInSnO/In} \mathrm{film,} \mathrm{and} \mathrm{clarifies} \mathrm{its} \mathrm{interface} \mathrm{characteris-}$ tics, biodegradable gelatin hydrogel/bioceramics composites for antitubercular drug delivery and tissue regeneration "Biodegradable polymer-coated, gelatin hydrogel/bioceramics ternary composites for antitubercular drug delivery and tissue regeneration," as well as the new preparation for the cancer treatment was offered "Preparation and characterization of folate targeting magnetic nanomedicine loaded with cisplatin".

In the current special issue were accepted also the papers on nanoporous contained polymer composites "Vinyl ester oligomer crosslinked porous polymers prepared via surfactantfree high internal phase emulsions," and "Effect of addition of soybean oil and gamma-ray cross-linking on the nanoporous HDPE membrane".

Sevan P. Davtyan

Aleksandr Berlin

Vladimir Agabekov

Nodari Lekishvili 

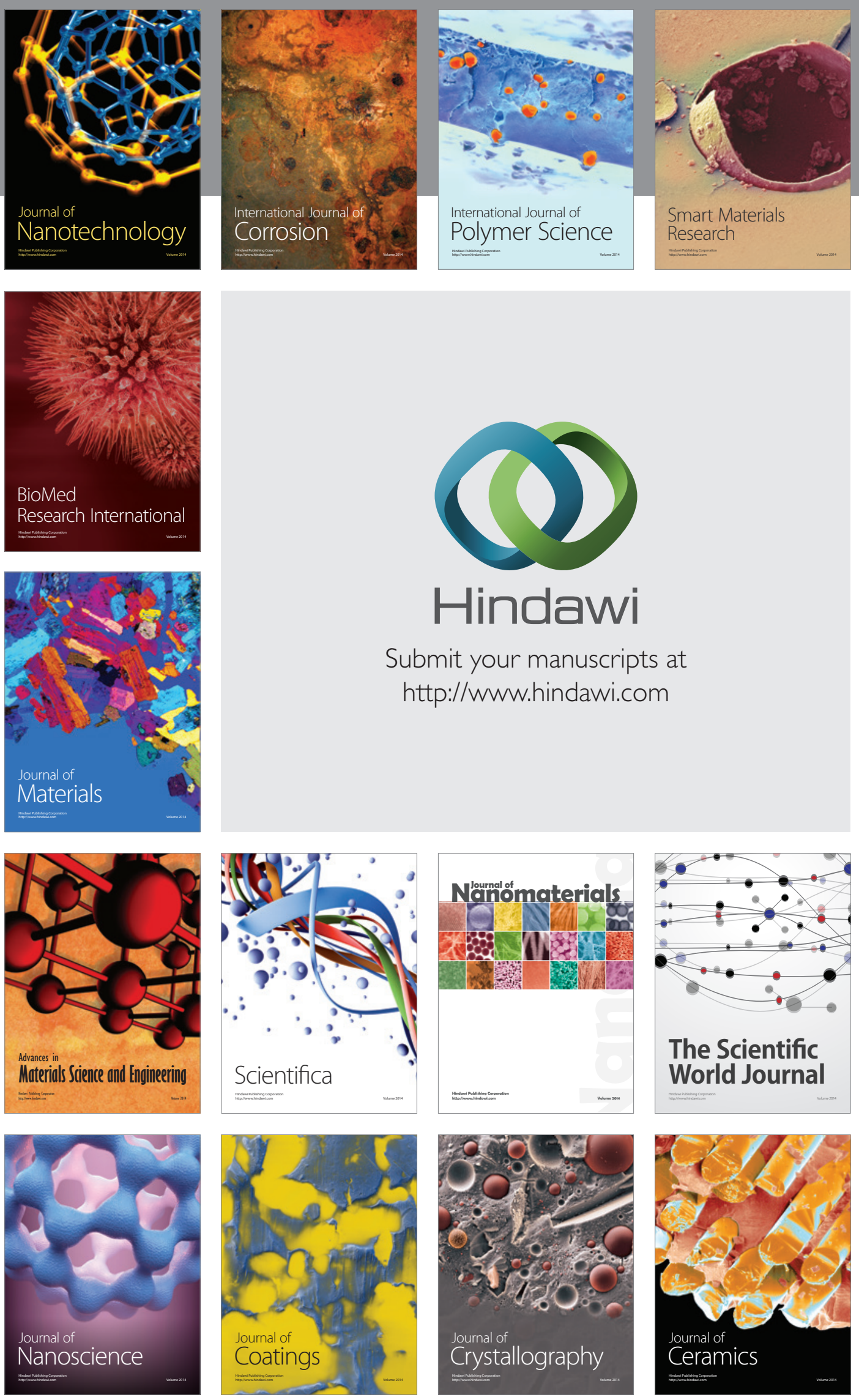

The Scientific World Journal

Submit your manuscripts at

http://www.hindawi.com

\section{World Journal}

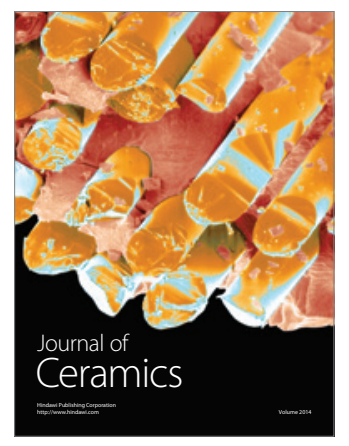

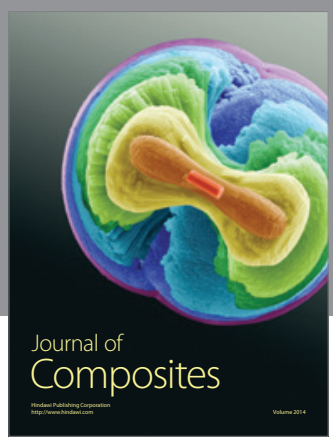
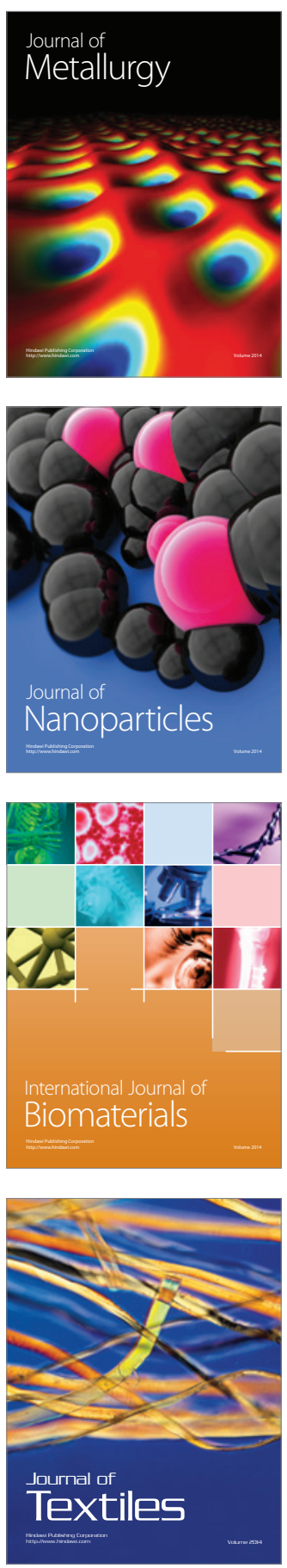